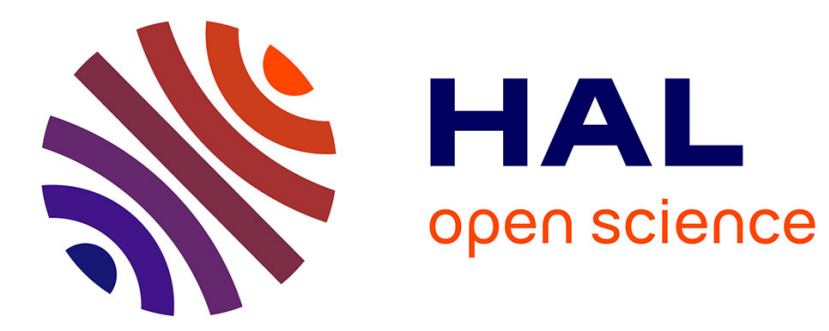

\title{
Science vs humanités : changer de modèle et de perception
}

Alexandre Moatti

\section{To cite this version:}

Alexandre Moatti. Science vs humanités: changer de modèle et de perception. Réalités industrielles. Annales des mines, 2016, Former pour l'inconnu, pp.28-32. halshs-01360376

\section{HAL Id: halshs-01360376 \\ https://shs.hal.science/halshs-01360376}

Submitted on 5 Sep 2016

HAL is a multi-disciplinary open access archive for the deposit and dissemination of scientific research documents, whether they are published or not. The documents may come from teaching and research institutions in France or abroad, or from public or private research centers.
L'archive ouverte pluridisciplinaire HAL, est destinée au dépôt et à la diffusion de documents scientifiques de niveau recherche, publiés ou non, émanant des établissements d'enseignement et de recherche français ou étrangers, des laboratoires publics ou privés. 


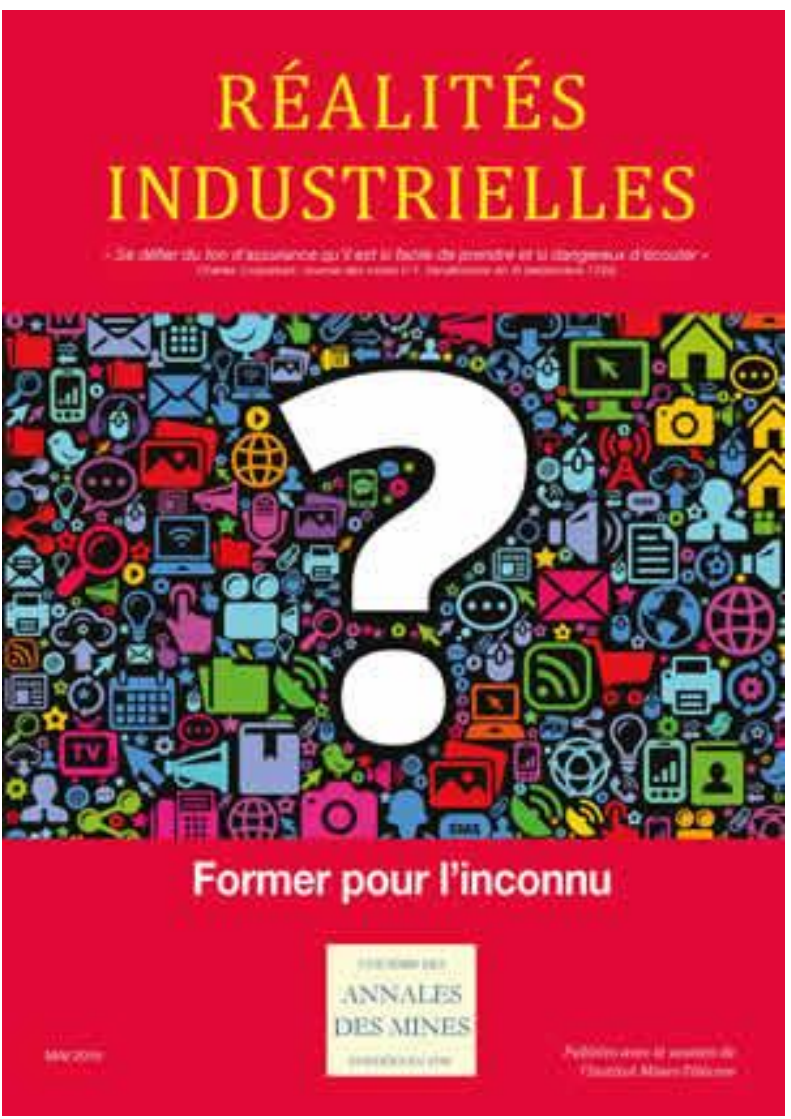

Editorial par Pierre Couveinhes,

Rédacteur en chef des Annales des Mines

Introduction par Marie-Josèphe Carrieu-Costa

Amble - Consultants

\section{Visions d'avenir pour l'éducation}

Comprendre et gérer la transition éducative

Par Philippe JAMET

et

Frédérique VINCENT

Institut Mines Télécom

La formation : une dynamique collective pour l'industrie Par Isabelle MARTIN

Secrétaire confédérale de la CFDT en charge des politiques industrielles et de la recherche 
Les STEM jobs (métiers scientifiques et technologiques) et le développement de l'industrie Par Christian MARGARIA

et

Bruno VERLON

Conseil général de l'Économie

Former pour l'avenir : le rôle joué par le programme Erasmus+

Par Elena TEGOVSKA

Direction générale Éducation et Culture, Commission européenne

Science vs humanités : changer de modèle et de perception

Par Alexandre MOATTI

Ingénieur en chef des Mines, chercheur associé à l'Université Paris-Diderot

La prospective : de l'anticipation à la préparation à l'avenir

Par Pierre PAPON

Physicien, professeur honoraire de physique thermique à l'École supérieure de physique et chimie industrielles de Paris (ESPCI)

\section{Quelques effets de la révolution numérique dans le domaine éducatif}

L'enseignement en ligne suscite une soif nouvelle de connaissance Par Gilles DOWEK

Chercheur à l'Inria et enseignant à l'École normale supérieure de Cachan

FUN, une plateforme de MOOCs au service des établissements d'enseignement supérieur Par Catherine MONGENET

Professeur d'université

De Lausanne à Yaoundé : l'aventure des MOOCs Pratiques et recherche sur les MOOCs, à Lausanne et en Afrique francophone

Par Dimitrios NOUKAKIS,

Gérard ESCHER

et

Patrick AEBISCHER

École polytechnique fédérale de Lausanne

\section{Enseigner et apprendre autrement}

Éducation et développement cognitif de l'enfant Par Olivier HOUDÉ

Professeur de psychologie à l'Université Sorbonne Paris Cité (USPC), directeur du LaPsyDÉ, UMR CNRS 8240 
Plaidoyer pour l'école primaire et l'apprentissage

Par Thierry WEIL

Professeur à Mines Paris Tech

L'artisanat augmenté Conceptions, enseignements et pratiques d'un art numérique appliqué aux métiers de l'artisanat

Par Bruno MONPÈRE

Directeur de la Chambre des Métiers et de l'Artisanat du Tarn-et-Garonne Cellule de re-

cherche@artisanumerique

Faire pour inventer l'avenir : une utopie concrète ?

Par Rafaële COSTE LARTIGOU

Inspectrice de l'Éducation nationale chargée de l'information et de l'orientation dans l'acadé-

mie de Versailles

et

Emmanuelle REILLE-BAUDRIN

Chercheur au CRTD-Cnam et directrice d'un centre d'information et d'orientation (académie de Créteil)

Comment approfondir les relations entre l'école et l'entreprise pour préparer les jeunes aux réalités économiques de demain ?

Par Christiane DEMONTÈS

Directrice de Centre d'Information et d'Orientation

Former pour l'inconnu : le rôle de la prospective

Par Thierry GAUDIN

Président de Prospective 2100

et

Anne-Marie BOUTIN

Présidente de l'APCI

\section{Hors dossier}

Deux cents ans de métallurgie à l’École des Mines de Saint-Étienne Par Michel DARRIEULAT

Ingénieur général des Mines honoraire

[Le texte qui suit est la " version auteur » de l'article paru dans Annales des Mines-Réalités industrielles (lien), mai 2016. Cette version, en date du 22 avril 2016, peut très légèrement différer de la version papier finalement publiée. A.M.] 


\section{Science vs. humanités : changer de modèle et de perception}

Alexandre Moatti, ingénieur en chef des Mines, chercheur associé à l'université Paris-Diderot.

Dans un précédent article (Annales des Mines, 2007)1, nous nous interrogions, parmi d'autres sujets, sur la place de la science dans l'enseignement secondaire, qui sert de moyen de sélection, souvent hélas indépendamment du corpus de connaissances qu'elle recouvre. Ceci reste vrai, mais nos interrogations ont évolué. Nous voudrions proposer ici quelques considérations sur le rôle historique et les conséquences actuelles de la sélection par la science; et sur la façon dont l'enseignement - dans un continuum allant du primaire au supérieur - pourrait être rééquilibré en faveur d'une diffusion assumée des humanités (notamment l'histoire) et de la culture générale.

Après 120 années de bons et loyaux services (1880-2000), la notion de sélection par la science doit être sérieusement reconsidérée : même si sur le terrain elle est déjà largement contournée, cette conception est encore très ancrée dans l'esprit de nos élites, dont une partie est le produit de cette sélection. Plus que séculaire, elle a été au fondement de l'école de la III République, celle de Jules Ferry et de Ferdinand Buisson, appuyée sur le positivisme de Comte et la science triomphante ; elle accompagnait les valeurs d'égalité républicaine, car la science était supposée plus "démocratique ", moins liée au milieu familial que la culture générale et littéraire. Elle a été intimement mêlée à l'idée de sélection méritocratique (le fameux « ascenseur social»), et bien sûr orientée en priorité vers la filière des classes préparatoires et des grandes écoles. L'ensemble voguait de conserve : plusieurs auteurs soulignent à raison que le système

\footnotetext{
1 "La diffusion de la culture scientifique, réalisations et réflexions », Réalités Industrielles (Annales des Mines), mai 2007, p. 54-59.
} 
de sélection par les mathématiques en France est conçu dès l'école primaire pour faire entrer $1 \%$ d'une classe d'âge à Polytechnique ou dans les écoles normales supérieures².

À l'heure où $85 \%$ d'une classe d'âge est amenée au baccalauréat, cette conception n'est plus viable. La démocratisation massive de l'enseignement (celle de Jules Ferry un siècle auparavant n'en était qu'une faible anticipation) a provoqué une inversion du paradigme : la question n'est plus « quel est le savoir qui permettra de trier les meilleurs ? (la réponse était : les mathématiques), elle est à présent « quel est le socle de connaissances minimal qu'on peut apporter à la globalité d'une classe d'âge jusqu'au baccalauréat? » Même si l'on ne saurait être d'accord avec les orientations utilitaristes (les savoirs « utiles » au détriment des savoirs fondamentaux) et issues du pédagogisme (comme les EPI enseignements pratiques interdisciplinaires dès la $5^{\mathrm{e}}$, sans base solide de connaissances par discipline) de la réforme Collège 2016, c'est bien ce changement de paradigme-là que cette réforme essaie d'accompagner, comme les multiples autres qui l'ont précédée.

Et même si ce n'est pas ouvertement affiché, la réforme actuelle vise aussi à réviser cette conception d'une suprématie de la sélection par la science (en fait : par les mathématiques, et ce dans le cadre d'un fort esprit d'abstraction qui s'étend aux autres sciences) - ce qui constitue un vrai enjeu. Cette sélection par la science, épine dorsale de notre enseignement secondaire pendant un siècle, laisse beaucoup trop d'élèves sur le bord de la route, dont l'esprit n'est adapté ni aux mathématiques ni à l'abstraction, et qui décrochent bien trop tôt. Ce n'est plus un système de sélection progressive mais d'exclusion précoce. Il était très finement réglé (instituteurs et professeurs du secondaire « signalaient » les élèves méritants, quel que soit leur milieu social, pour les orienter vers cette filière scientifique) jusque dans les années 1970 - cette réelle méritocratie a ensuite poursuivi sur son erre. En 1980, 25\% d'une classe d'âge soit 220000 élèves $^{3}$ obtenait le baccalauréat ; quand ces chiffres sont triplés ${ }^{4}$ (en 2015, on

\footnotetext{
${ }^{2}$ Voir par exemple J.-M. Lévy-Leblond, L'Esprit de sel. Science, Culture, Politique, Fayard, 1981 : « En un raccourci à peine outré, on peut affirmer que les programmes de l'École polytechnique déterminent ceux de l'école maternelle [...] par ce mécanisme téléologique, les besoins assignés à l'infime minorité qui émerge au sommet de la pyramide éducative imposent leurs règles à la vaste majorité de ceux qui la quittent aux divers paliers intermédiaires. " Étonnamment, on peut remonter cette idée à travers l'histoire, par exemple chez l'historien Marc Bloch (1886-1944): "Tous nos programmes scientifiques d'enseignement secondaire, me disait un physicien, sont conçus en vue de celui de Polytechnique " (M. Bloch, "Sur la réforme de l'enseignement ", note rédigée pour les Cahiers politiques, 1944, reprise dans L'Étrange Défaite, Folio Histoire $n^{\circ} 27$ ).

${ }^{3}$ http://media.education.gouv.fr/file/06 juin/19/3/Baccalaureat-tableaux-statistiques 61193.pdf

${ }^{4}$ http://www.education.gouv.fr/cid56455/resultats-du-baccalaureat-session-de-juin-2015.html
} 
compte 620000 bacheliers soit 77\% de la classe d'âge), l'idée même de sélection n'est plus adaptée, elle est antinomique voire contre-productive. Démocratie ne rime plus avec méritocratie.

Tout ceci est sans doute évident dans les esprits, depuis une bonne quinzaine d'années. Mais il convient néanmoins d'aller jusqu'au bout de la plaidoirie, car une élite dirigeante qui est issue de la sélection par la science ne semble pas avoir perçu son reflux, nécessaire et effectif. Cette sélection reste soutenue par deux centres de pouvoir élitaires sans grand rapport entre eux (presque à l'opposé l'un de l'autre) mais se rejoignant sur ce point. D'un côté, le milieu normalien (avec l'influence qu'il peut avoir au sein de l'Éducation nationale - par exemple chez les inspecteurs généraux et professeurs de mathématiques) : il s'agit, depuis 1880 et la prise de pouvoir de l'ENS sur Polytechnique dans la suprématie scientifique, de tout conserver à l'identique pour que non pas $10^{-3}$ mais $10^{-7}$ d'une classe d'âge puisse obtenir la convoitée médaille Fields, et ainsi maintenir le rang de la France de deuxième puissance mondiale en mathématiques, après les États-Unis. De l'autre côté, un milieu polytechnicien et des Grands Corps d'État, très conservateur, qui n'imagine pas que le système de sélection puisse être différent de celui qui a fait réussir ses membres, et qui reste ancré dans la réminiscence saint-simonienne d'une technocratie, i.e. d'un gouvernement par la technique et par la rationalité, appuyé sur une formation et une sélection par la science ${ }^{5}$.

Cependant la société est plastique et, on l'a dit, les lignes ont bougé depuis vingt ans, indépendamment de la vision des élites dirigeantes. La fabrique des élites s'est déplacée : qu'on le veuille ou non, qu'on le déplore ou non, une France d'ingénieurs sélectionnés par la science - s'efface, en même temps que s'efface le tissu industriel ancien, au profit d'un autre type d'élites, nouvelle génération de dirigeants formés par les écoles de commerce, par SciencesPo ou par l'ENA. Un autre signe est l'amorce d'une fin de prééminence de la section $S$ (sciences) en 1 $1^{\mathrm{e}}$-Terminale : les sections $\mathrm{L}$ et ES sont à présent mieux considérées qu'elles ne l'étaient - notamment pour l'accès aux

\footnotetext{
${ }^{5}$ " L’École polytechnique, cette école militaire qui forme par la science des hauts fonctionnaires qui veulent devenir chefs d'entreprise. Trop de contradictions ", avions-nous tweeté (24 décembre 2015) à propos des graves problèmes posés par la réforme de I'X annoncée mi-décembre - notamment quant à son lien avec l'université. Sur la tradition élitaire saint-simonienne en France, voir A. Moatti, "La figure de Saint-Simon (1760-1825) dans les discours technocratiques français ", colloque Université Technologique Belfort-Montbéliard, mars 2016, en ligne sur $\underline{\mathrm{HAL}-\mathrm{SHS}}$.
} 
grandes écoles commerciales. C'est d'ailleurs une conséquence du glissement évoqué des élites, auquel s'adaptent notamment les stratégies parentales de contournement. Mais qu'on ne se leurre pas : cette nouvelle modalité de sélection des élites continue à s'appuyer sur les mathématiques ${ }^{6}$; de plus elle reste terriblement élitaire, s'étant lovée, tout en les accentuant, dans les défauts du système de sélection par les grandes écoles et par la science : elle se fait sur concours, de manière plus précoce (maints élèves suivent une coûteuse préparation pendant leur année de Terminale !) - les échecs étant eux aussi plus précoces -, moins égalitaire, et enfin au bénéfice de milieux sociaux bien informés ${ }^{7}$. Elle contribue par défaut à une spécialisation, ou à un choix fait de plus en plus jeune (sinon un défaut de choix : si l'élève est bon en maths, il continue à... avoir le choix ; sinon, il doit faire son choix). C'est aussi contre cette spécialisation qu'il faut lutter, en conservant des horizons d'éducation les plus larges et d'orientation les plus ouverts possibles.

Ceci nous amène à notre second point, la nécessité de repenser la conception commune que nous avons de notre enseignement: il s'agit d'avoir pour principe fondateur non plus celui de la sélection par la science, mais celui d'un enseignement le plus large possible d'humanités et de culture générale, dans le secondaire comme en premier cycle de l'enseignement supérieur - qui bien sûr n'exclue pas les sciences. Plus que les humanités antiques (à savoir l'enseignement du grec et du latin), c'est bien l'histoire (dont l'histoire de la littérature ou de la philosophie, l'histoire des sciences et l'épistémologie) qu'il s'agit de réhabiliter, et à travers elle l'accès à une culture générale commune. Certes, cette notion de culture générale est peu en vogue actuellement - elle se heurte, quasi par définition, au communautarisme et à toute espèce de revendication identitaire, chacun voulant écrire sa propre histoire.

Pourtant, déjà en 1945, l'historien Marc Bloch appelait l'enseignement à « un examen de conscience » et à un « plan de rénovation ». Comparant l'enseignement de l'histoire à celui des sciences - ceci reste d'actualité et correspond à notre propos -, il

\footnotetext{
${ }^{6}$ Ce qui est maintenant demandé en probabilité/statistiques en classes préparatoires commerciales (type ECS) est de niveau plus difficile que les mathématiques de classes préparatoires scientifiques (type MPSI-PCSI), pour des élèves qui n'utiliseront plus aucun de ces concepts mathématiques un an plus tard.

${ }^{7}$ C'est, de nos jours, une manifestation significative de ce que le sociologue Éric Maurin a appelé Le Ghetto français (Seuil 2004), celui des élites et de leur autoreproduction.
} 
écrivait : «Là encore, comme dans les sciences physiques, un choix neuf s'impose. » Quelles sont ces bases sur lesquelles un enseignement d'humanités et d'histoire pourrait être « refondu » (selon son expression), en vue d'une culture générale et de valeurs communes? Nous nous attacherons moins aux contenus (beaucoup d'encre a coulé à ce propos lors du débat Collège 2016) qu'à la méthode - après tout, une méthode scientifique pourrait être mieux mise en valeur en histoire et en culture générale.

\section{Esprit de Marc Bloch, es-tu là ?}

Le texte de 1944 de l'historien Marc Bloch (repris dans L'Étrange Défaite, Folio Histoire $n^{\circ} 27$ ) est d'une surprenante actualité ${ }^{8}$.

Sur les ressources budgétaires : "Il faut que la France de demain sache dépenser incomparablement plus qu'elle ne s'y est résignée jusqu'ici. »; à ce propos Bloch souligne le déplorable état des bibliothèques universitaires.

Sur les gouvernants français : "Le peu d'intérêt qu'ils portent aux choses de l'esprit. »

Sur le baccalauréat : "Certaines condamnations à mort s'imposent. Qui croit encore au baccalauréat, à la valeur de choix, à l'efficacité intellectuelle de cette aléatoire forcerie?»

Sur les grandes écoles : il faut persuader les « anciens élèves des grandes écoles que ces établissements parés de tous les prestiges du souvenir et de la camaraderie doivent être supprimés ».

Sur la création de l'ENA après-guerre : les gouvernants « préférèrent tracer le plan d'une nouvelle école spéciale : une autre École des sciences politiques, encore un peu mieux close que sa rivale ».

Sur les sciences humaines : "Qu'est-ce qu'une Faculté de Lettres, sinon, avant tout, une usine à fabriquer des professeurs $[\ldots]$ ? »

Il conviendrait par exemple de « montrer aux élèves que l'histoire est d'abord une enquête $[\ldots]$; si on leur apprend à dater un document [...], si on leur explique comment on parvient à avoir une connaissance approfondie des civilisations, on les associe à la recherche de la vérité ${ }^{9} \gg$. Ces humanités nouvelles pourraient aussi, dès le secondaire, coupler à l'histoire un peu d'anthropologie ou d'ethnologie : ces dernières disciplines ont su développer une méthodologie scientifique, fondée sur des faits et des observations, qui les ont préservées des travers dans lesquels sont tombés des pans entiers d'autres disciplines relevant des "sciences humaines et sociales », comme

\footnotetext{
${ }^{8}$ Assez bref, il mériterait d'être republié dans son intégralité dans ce numéro des Annales des Mines « Former pour l'inconnu ? ». On peut le trouver en ligne à http://www.marcbloch.fr/notes.html.

${ }^{9}$ Yves Michaud, Philosophie Magazine, mensuel n96, février 2016, p. 66.
} 
l'économie ou la sociologie - en partie récupérées et marchandisées dans le cadre d'un utilitarisme de court terme ${ }^{10}$, auquel s'oppose toute idée de culture générale. Troisième piste, y associer l'histoire des idées et la référence contemporaine qu'elle peut induire. L'enseignement de l'histoire n'est ni un récit national (position « conservatrice » émise lors du récent débat à propos de Collège 2016) ni une pure mise en contexte dans une époque et un milieu culturel donnés (position «postmoderne ») : l’histoire des idées avec ses répétitions d'arguments au cours des âges, y compris de nos jours - aide à comprendre le monde contemporain et à y exercer sa capacité de discernement. Bien évidemment les choix de contenus seront toujours subjectifs ${ }^{11}$ : mais la mise en évidence d'une méthode scientifique et la mise en culture relative à notre contemporanéité sont deux axes directeurs permettant d'intéresser l'élève et de l'aider à décrypter le monde.

Le cas de l'enseignement supérieur se pose avec autant sinon plus d'acuité. Là aussi, remontons aux sources de la III République : c'est en 1872 qu'Émile Boutmy crée l'École libre des sciences politiques (maintenant SciencesPo) ; à la suite de la défaite de 1870, fasciné par la puissance intellectuelle de l'Allemagne, il estimait que l'université française avait failli à sa tâche en matière d'humanités. Il faut, à nouveau, innover dans l'enseignement supérieur et, à nouveau, en s'appuyant sur les humanités et la culture générale. Exercer chez l'étudiant un regard critique - au sens le plus positif du terme, celui du discernement, de la mise en éveil : écouter et comprendre ce qui se dit d'intéressant à gauche, à droite, tous azimuts, et en dégager sa propre opinion. Vive le débat intellectuel !, certes, mais encore faut-il avoir quelques bases solides pour y participer ou le comprendre avec profit. En ce sens, la notion contemporaine d'humanités peut mutatis mutandis être comparée à celle d'avant ce que Julien Benda a appelée en 1927 La Trahison des clercs, c'est-à-dire la participation des « intellectuels » (nouvelle catégorie à l'époque) au débat public, au détriment du travail d'érudition et

\footnotetext{
${ }^{10}$ Nous remercions Marie-Josèphe Carrieu-Costa, coordinatrice du présent numéro, des idées échangées par courriel à ce propos.

${ }^{11}$ Par exemple, sachant que tout choix est subjectif et discutable - mais les exemples sont nécessaires : I'histoire oubliée du jansénisme et de ses valeurs (en illustration d'une réflexion sur l'histoire des religions, ou sur les rapports entre pouvoir et religion) ; l'histoire des utopies sociales pré-marxiennes comme le saint-simonisme (en illustration de la seconde révolution industrielle et de la naissance de la III République mentionnée ci-dessus) ; I'histoire de la critique de la modernité, de Rousseau à Ellul (en décryptage de la critique contemporaine de l'internet) ; l'histoire de la fondation et des principes de l'enseignement laïque en France entre 1880 et 1900.
} 
de connaissance - celui des humanités, justement : histoire, études littéraires, philosophie.

Poursuivons sur ce plan politique, celui des affaires de la cité et de la conception commune d'une vie en société. Les formations en sciences humaines et sociales et en littérature sont souvent les parents pauvres d'une formation universitaire qui l'est déjà elle-même, ne correspondant pas à la voie royale des grandes écoles ni à la voie moins royale mais très fréquentée des formations spécialisantes. Elles visent souvent à reproduire un modèle de débouchés vers l'enseignement-recherche - comme le notait déjà Bloch en 1944 ; elles regroupent dans diverses sous-catégories des enseignements trop spécifiques, trop politisés, parfois dénigrés par les étudiants eux-mêmes. Ce n’est pas le moindre des paradoxes de l'enseignement universitaire français d'être pris entre deux feux, d'avoir suivi deux tendances en apparence contradictoires : d'un côté, une forme de sclérose idéologique et de l'autre une marchandisation utilitariste de type néo-libéral, toutes deux déconnectées de la transmission durable des savoirs.

Signal positif à bas bruit à nouveau, certains départements d'université se sont avisés de sortir de ce dilemme, en prenant la peine de réfléchir et d'entamer une mutation importante ${ }^{12}$. Ils se sont aussi avisés que de plus en plus, les entreprises pouvaient être intéressées par des profils différents des profils scientifiques, plus cultivés, plus créatifs. Comme le souligne J.-F. Pradeau, l'enseignement supérieur prend progressivement conscience « d'un besoin exprimé à la fois par les étudiants, désireux de s'instruire indépendamment des enseignements professionnalisants, et par les employeurs », ces derniers s'intéressant à recruter « des jeunes gens qui ont une culture historique et littéraire [...], une forme d'érudition qui va leur permettre de mieux comprendre le monde qui les entoure et de mieux agir ${ }^{13}{ }$. Quant à nous, notre feuille de route, à notre échelle, est de participer à redonner leurs lettres de noblesse aux humanités via le numérique : notre projet est la création d'une chaîne vidéo de

\footnotetext{
${ }^{12}$ On citera par exemple, sans que cette liste soit exhaustive, la formation CPES (Cycle pluridisciplinaire d'études supérieures), qui reste très élitaire, du pôle Paris Sciences \& Lettres (PSL) ; les licences " Humanités » ou "Sciences \& Humanités » des universités Paris XI-Nanterre, Aix-Marseille, ou de l'université technologique de Compiègne. Une discussion sur ces nouvelles formations figure (p. 17 et ss) dans J.-F. Pradeau, "L'avenir des humanités dans l'entreprise ", Carnets de l'Institut Diderot, 2011 (en ligne).

13 J.-F. Pradeau, op. cit., p. 23.
} 
conférences d'humanités et de culture générale, à rattacher à une institution publique ${ }^{14}$.

\section{Le « numérique éducatif » : ni cet excès $d$ 'honneur ni cette indignité !}

Secondaire par rapport aux deux sujets qui nous occupent, il ne mérite sans doute ni cet excès d'honneur ni cette indignité. L'excès d'honneur, c'est celui des trompettes du "numérique à l'école » (toujours ré-embouchées à l'occasion, et quasiment toujours à l'identique depuis une bonne quinzaine d'années) : elles peuvent être considérées comme un exemple de «solutionnisme ${ }^{15}$ » - le numérique venant à être présenté, à l'appui de la réforme en cours du collège, comme allant résoudre tous les problèmes de l'Éducation nationale. Il faudrait aussi parler de certains formats vidéos, de type TED ou TEDx, certes plaisants en apparence mais aux interventions souvent peu approfondies, dont les thèmes (le bien-être, etc.) s'éloignent de la diffusion des savoirs, et qui souvent véhiculent une idéologie technoprophétique « solutionniste » (« vous allez voir ce que vous allez voir, cela ira mieux demain, grâce à la technique, grâce à moi,... ») ${ }^{16}$. Inversement, l'opprobre portée sur le numérique éducatif est souvent exagérée : que ce soit la critique tous azimuts de l'encyclopédie Wikipédia, ou l'épouvantail d'une mutation anthropologique liée aux nouvelles manières d'apprendre (ceux qui en parlent s'y connaissent peu, et vice-versa), ou encore le spectre d'une décérébration - «leurs tablets sont des tabula rasa» croit savoir de manière amusante mais peu étayée le philosophe allemand Roland Reu $\beta^{17}$, parlant des générations qui le suivent.

Finalement, on ne peut qu'être frappé par la trop grande longévité de nos modèles d'éducation publique en France, associée à leur faible capacité de remise en question: les grands Corps d'État depuis l'Ancien Régime; les grandes écoles scientifiques (et la sélection par la science qu'elles ont induite) depuis la Révolution et l'Empire; la filière de sélection via les sciences politiques et les grandes écoles

\footnotetext{
${ }^{14}$ En continuité de ce que nous avons fait avec science.gouv.fr (2004-2014), et bibnum.education.fr (depuis 2008), bibliothèque numérique d'histoire des sciences (MESR et Fondation Maison des Sciences de l'Homme, avec le soutien de la Fondation de l'École polytechnique).

${ }^{15}$ Ce concept a été introduit par l'essayiste américain Evgeny Morozov. Cette même notion avait été introduite de manière bien plus approfondie et intéressante par le philosophe Jacques Ellul (1912-1994), par exemple dans Le Bluff technologique, 1988 ("La technique nous est dorénavant présentée comme la seule solution à tous nos problèmes collectifs »).

${ }^{16}$ Une critique bienvenue des conférences TED est parue dans The Guardian, "We need to talk about TED », par Benjamin Bratton, 30 décembre 2013 (http://www.theguardian.com/commentisfree/2013/dec/30/we-need-totalk-about-ted). Une critique des MOOC après quelques années d'existence serait elle aussi bienvenue.

${ }^{17}$ R. Reuß, Sortir de l'hypnose du numérique, Strömfeld 2012, trad. fcse Des Îlots de Résistance, 2013.
} 
commerciales depuis le début de la III République. Ces modèles très élitaires ont atteint leurs limites, nous l'avons dit. Bien au-delà du seul milieu de l'Éducation nationale, une prise de conscience et une action rapide des élites qu'ils ont formées est nécessaire afin de modifier en profondeur le système : en auront-elles la vision, et la volonté?

Mais ce qui frappe plus encore, à l'examen sur une certaine période de temps, c'est que le diagnostic et les propositions portés ici (fin d'une sélection par la science, refondation par les humanités) l'avaient été il y a longtemps déjà : il n'est pas banal que l'article de 1944 de Marc Bloch n'ait pas pris une ride, et même résonne avec une pertinence et une acuité renouvelées. Loin d'un nostalgique retour en arrière, c'est un signe à considérer positivement, montrant que l'histoire (ici celle de la réflexion sur l'éducation) éclaire le présent : le programme est tracé de longue date, il convient de le suivre. Il ne s'agit pas d'opposer sciences et humanités, ni de se passer des sciences, mais de prendre acte que le système de sélection, que ce soit par les mathématiques ou les "sciences politiques", n'est plus du tout un modèle à défendre comme un étendard. Que cet étendard doit être à présent celui de la culture générale, de l'histoire et de sa mise en perspective, à considérer comme méthode éducative, permettant de faire société. Pour terminer par une pirouette, en empruntant aux métaphores de Montaigne et de Pascal, souvent filées mais rarement mêlées : à l'esprit de géométrie d'une tête (trop) bien faite, sans doute faut-il préférer l'esprit de finesse émanant d'une tête bien pleine.

(sur la page de description des auteurs)

\section{MOATTI Alexandre}

Ingénieur en chef des Mines (Conseil général de l'économie), Alexandre Moatti (www.moatti.net) est chercheur associé à l'université Paris VII-Denis Diderot (laboratoire SPHERE UMR 7219). II participe à de nombreuses actions publiques de diffusion de la connaissance depuis 2004 (p. ex. www.bibnum.education.fr). II a été délégué de la Fondation CGénial (culture scientifique dans les lycées et collèges de 2007 à 2012) - il en est administrateur depuis. II est par ailleurs président du jury d'admission de l'ENSCI (École nationale supérieure de Création industrielle). 